\title{
Conceptual theories in structural dynamics
}

\begin{abstract}
This book discusses the conceptual theory of structural dynamics, using simplified methods and clear, concise explanations. It illustrates all the hypotheses in a simple and effective way and describes in detail the derivation of all related formulations. Further, comprehensive stepby-step explanations combined with conceptual derivations, drawings and figures allow readers to grasp all the analytical formulations related to the dynamics of structures. Covering free and forced vibrations of single- and multi-degree of freedom systems represented as structure, subjected to dynamic load, the book also explores the most common types of dynamic loads applicable to structures, such as harmonic loads, impact loads and earthquakes, presenting relevant details, derivations and effective problems to explain the concept for various conditions. In addition, each chapter provides examples at different levels to help students, researchers and engineers gain a better understanding of the topics better, and includes numerous real-world problems to familiarize readers with the challenges related to structural engineering.
\end{abstract}

